\title{
SÍNTESE DE AMIDAS E SULFONAMIDAS DE $\beta$-D-GALACTOPIRANOSILAMINA E $\beta$-LACTOSILAMINA E AVALIAÇÃO DE SUAS INTERAÇÕES COM LECTINAS DE Erythrina cristagalli E DE Ricinus communis
}

\author{
Anna Paola Butera e José Dias de Souza Filho \\ Departamento de Química, Instituto de Ciências Exatas, Universidade Federal de Minas Gerais, Av. Presidente Antônio Carlos, \\ 6627, 31270-901 Belo Horizonte - MG, Brasil \\ Diogo Teixeira Carvalho, Rute Cunha Figueiredo, Luiz Carlos Alves de Faria, Maria Angélica Nunes, Maria Auxiliadora \\ Fontes Prado e Ricardo José Alves* \\ Departamento de Produtos Farmacêuticos, Faculdade de Farmácia, Universidade Federal de Minas Gerais, Av. Presidente \\ Antônio Carlos, 6627, 31270-901 Belo Horizonte - MG, Brasil \\ Milton Hércules Guerra de Andrade e Karina Taciana Santos Silva \\ Departamento de Ciências Biológicas, Instituto de Ciências Exatas e Biológicas, Universidade Federal de Ouro Preto, Rua \\ Diogo de Vasconcelos, 122, 35400-000 Ouro Preto - MG, Brasil \\ Recebido em 5/10/06; aceito em 18/12/06; publicado na web em 17/7/07

\begin{abstract}
SYNTHESIS OF AMIDES AND SULFONAMIDES OF $\beta$-D-GALACTOPYRANOSYLAMINE AND $\beta$-LACTOSYLAMINE AND EVALUATION OF THEIR INTERACTIONS WITH THE LECTINS FROM Erythrina cristagalli AND Ricinus communis. We report herein the synthesis of some $\beta$-D-galactopyranosylamine and $\beta$-lactosylamine amides and sulfonamides. The interactions of these compounds with lectins from the seeds of Erythrina cristagalli (LEC) and Ricinus communis (RCA120) were evaluated in a hemagglutination inhibitory activity assay. D-Galactose and lactose were used as reference compounds. The $\beta$-lactosylamine amides and sulfonamides were nearly as active as lactose in inhibiting LEC mediated hemagglutination and were less active against RCA120 agglutinin. The $\beta$-D-galactopyranosylamine amides and sulfonamides were, with one exception, considerably less active than D-
\end{abstract} \\ galactose in the assay with both lectins.
}

Keywords: carbohydrates; plant lectins; amides.

\section{INTRODUÇÃO}

Lectinas são proteínas desprovidas de atividade enzimática que se ligam de maneira reversível e específica a carboidratos. Distinguem-se das imunoglobulinas, que reconhecem especificamente antígenos, eventualmente sacarídicos, seja estruturalmente, seja porque estas últimas dependem de estímulo antigênico para serem sintetizadas. Essa classe de proteínas estruturalmente diversificada é amplamente distribuída na natureza e, em geral, está relacionada a processos de reconhecimento celular mediados por suas interações com carboidratos ${ }^{1-4}$. É reconhecida a participação destas interações em processos fisiológicos e patológicos importantes, tais como no controle do tráfego intracelular de glicoproteínas, na adesão de agentes infecciosos à célula hospedeira, nas interações do sistema imune e na metástase de tumores ${ }^{5}$. A seletividade das interações entre lectinas e carboidratos teve notável importância histórica pela contribuição na descoberta dos grupos sangüíneos humanos ${ }^{6}$. Em vista do exposto, as lectinas têm sido consideradas potenciais alvos moleculares de substâncias com atividades antitumoral e antiparasitária ${ }^{7,8}$. Denota-se a seletividade pela distinção, por parte da lectina, entre ligantes monossacarídeos, sendo comum a classificação das lectinas de acordo com o monossacarídeo pelo qual exibe maior afinidade, normalmente aqueles presentes em células eucarióticas: D-manose, D-galactose $/ N$-acetil-D-galactosamina, $\mathrm{N}$ acetil-D-glicosamina, L-fucose e ácido $N$-acetil-D-neuramínico ${ }^{5}$.

O ensaio de hemaglutinação apresenta-se como uma das técnicas mais simples de avaliação das interações entre carboidratos e lectinas. Seu vasto emprego deve-se à facilidade de obtenção dos

*e-mail: ricardodylan@farmacia.ufmg.br eritrócitos do tecido sangüíneo e à riqueza qualitativa dos resíduos sacarídicos que são expressos em suas superfícies. Existem diversos estudos em que a propriedade aglutinante das lectinas é utilizada para a obtenção de dados da composição sacarídica da superfície de outros tipos celulares, como por ex., de células tumorais ${ }^{1,9,10}$. A aglutinação celular promovida por lectinas é possível, uma vez que, estas, em sua maioria, são oligoméricas e multivalentes. As lectinas vegetais e as galectinas, lectinas animais especificas para D-galactose, são particularmente aglutinantes por apresentarem os sítios de interação com carboidratos posicionados nas extremidades opostas dos oligômeros ${ }^{5}$.

As lectinas vegetais foram as primeiras a serem descobertas e seu fácil isolamento, comparado ao de outras fontes, faz com que, ainda hoje, sejam muito estudadas e caracterizadas em grande número ${ }^{4}$. As lectinas isoladas de espécies vegetais são especialmente utilizadas como sistema modelo para o estudo das bases moleculares do reconhecimento lectina-carboidrato pois, apesar da semelhança da estrutura primária destas proteínas, suas especificidades a carboidratos podem ser diferentes. Até o momento, as estruturas tridimencionais de cerca de 60 lectinas vegetais foram elucidadas por cristalografia de raios $\mathrm{X}$, nas formas livres e complexadas com uma variedade de compostos ligantes ${ }^{11,12}$.

A lectina isolada das sementes de Erythrina cristagalli (LEC) apresenta seletividade pelos monossacarídeos D-galactose e $\mathrm{N}$-acetilD-galactosamina ${ }^{13}$. Como ocorre em outras lectinas de fabáceas, o sítio de ligação a carboidratos apresenta-se como uma depressão na superfície molecular. Esta lectina apresenta estrutura homodimérica, sendo que cada domínio contém um sítio de ligação a carboidrato, além de dois sítios de interação para íons, um para $\mathrm{Ca}^{2+}$ e um para $\mathrm{Mn}^{2+}$, ambos necessários para interação com carboidratos ${ }^{14}$. 
Das sementes de Ricinus communis (Euphorbiaceae) são isoladas a ricina, proteína com fraca atividade hemaglutinante, altamente citotóxica, também denominada RCA60 (60 kDa), e a aglutinina RCA120 (120 kDa), não citotóxica. A RCA60 é constituída por duas cadeias polipeptídicas interligadas por ponte dissulfeto. A cadeia A é uma RNA-N-glicosidase capaz de inibir o processo de síntese protéica em células de mamíferos, ao remover um resíduo de adenina do RNA ribossomal. Já a cadeia B é uma lectina, que tem seletividade por $\beta$-D-galactosídeos. É responsável pelos processos de adesão celular da toxina e de sua translocação citoplasmática até os ribossomos. A aglutinina RCA120 é um tetrâmero composto por duas cadeias A' e B'. As cadeias polipeptídicas de RCA60 e RCA120 são estruturalmente relacionadas. Apresentam $93 \%$ e $84 \%$ de homologia entre as sequiências primárias das cadeias A e B, respectivamente. No entanto, as lectinas apresentam especifidades diferentes para a ligação com monossacarídeos, oligossacarídeos e glicopeptídeos. Assim como LEC, as lectinas de $R$. communis apresentam afinidade pelo dissacarídeo lactose superior à da D-galactose ${ }^{14,15}$.

No presente trabalho é relatada a síntese de derivados de Dgalactose (1) e de lactose (2) e a investigação das diferenças nas interações destes derivados com lectinas isoladas das sementes de Erythrina cristagalli (LEC) e de Ricinus communis, especificamente a aglutinina RCA120, pela comparação da capacidade inibitória da hemaglutinação desses derivados em relação a $\mathbf{1}$ e $\mathbf{2}$.
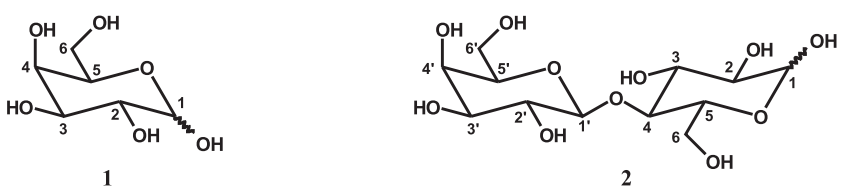

Teve-se como objetivo central a identificação de ligantes sintéticos, monovalentes, com maior afinidade por essas lectinas, com vistas ao desenvolvimento de inibidores de suas interações com ligantes naturais. Sabe-se que, de modo geral, as constantes de associação individuais entre lectinas e monossacarídeos não são muito elevadas ${ }^{16}$. A afinidade é potencializada nas interações com oligossacarídeos, para as quais os valores de constantes de associação são até mil vezes maiores que para os monossacarídeos ${ }^{5}$. O planejamento de ligantes monovalentes foi fundamentado na modificação de C-1, com a introdução de substituintes contendo grupos aceptores e doadores de ligação de hidrogênio, além de grupos hidrofóbicos. Tais substituintes poderiam interferir nos processos de solvatação e dessolvatação dos ligantes e de seu sítio de interação, aumentando a afinidade carboidratolectina ${ }^{17}$. Essa posição é de fácil manipulação química, além de ser bastante utilizada para a síntese de ligantes mono- e polivalentes de lectinas ${ }^{1}$. Assim, utilizando-se reações clássicas da química de carboidratos foram sintetizados cinco derivados da D-galactose (13ae) e quatro derivados da lactose (14a-d) que contêm grupos amido e sulfonamido ligados ao carbono anomérico (C-1) (Figura 1).

É importante ressaltar que os grupos amido e sulfonamido dos compostos planejados preservam a capacidade de formar ligações

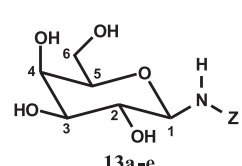

13a-c

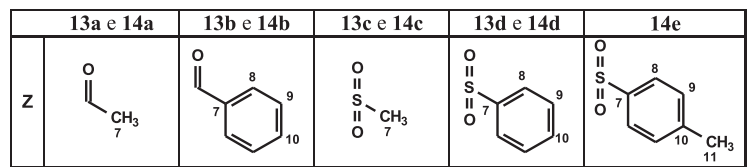

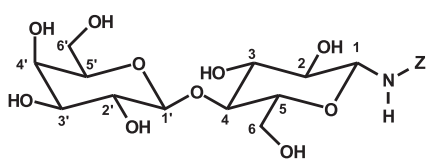

14a-d
Figura 1. Derivados da D-galactose e da lactose sintetizados de hidrogênio com o sítio de interação das lectinas, mas não apresentam a instabilidade indesejada de glicosilaminas ${ }^{18}$. As glicosilaminas são reconhecidamente instáveis, susceptíveis a reações de anomerização e de hidrólise ${ }^{17}$, que envolvem a formação de um intermediário imínio (Figura 2). A formação do íon imínio é menos favorecida nas amidas e sulfonamidas anoméricas, devido a menor basicidade do nitrogênio nesses compostos.

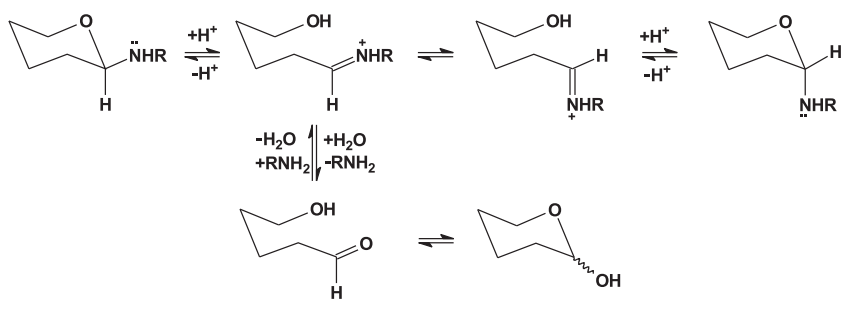

Figura 2. Reações de anomerização e hidrólise de glicosilaminas

\section{RESULTADOS E DISCUSSÃO}

\section{Síntese}

As amidas e sulfonamidas 13a-e e 14a-d foram sintetizadas empregando-se metodologias clássicas da química de carboidratos, de acordo com o esquema de síntese mostrado na Figura 3.
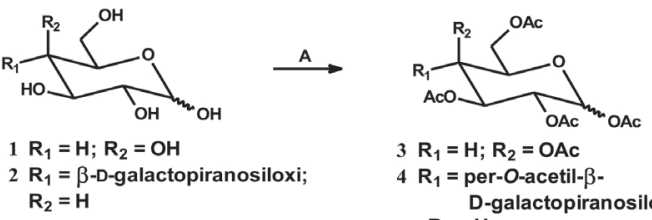

$3 \mathbf{R}_{1}=\mathrm{H} ; \mathbf{R}_{2}=\mathrm{OAC}$

$4 \mathrm{R}_{1}=$ per-O-acetil- $\beta$ D-galactopiranosiloxi;

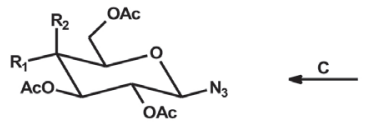

$7 \mathrm{R}_{1}=\mathrm{H} ; \mathrm{R}_{2}=\mathrm{OAC}$

$8 R_{1}=$ per-O-acetil- $\beta$ $\mathbf{R}_{\mathbf{2}}=\mathbf{H}$-galactopiranosiloxi;

$\downarrow$

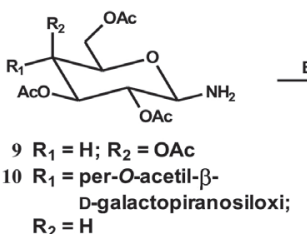

$\mathbf{R}_{\mathbf{2}}=\mathbf{H}$

\begin{tabular}{ccc}
\hline \multicolumn{2}{c}{ DERIVADO } & $\mathrm{Z}$ \\
\hline $11 \mathrm{a}$ e 12a & $13 \mathrm{a}$ e 14a & $\mathrm{COCH}_{3}$ \\
$11 \mathrm{~b}$ e 12b & $13 \mathrm{~b}$ e 14b & $\mathrm{COC}_{6} \mathrm{H}_{5}$ \\
$11 \mathrm{c}$ e 12c & $13 \mathrm{c}$ e 14c & $\mathrm{SO}_{2} \mathrm{CH}_{3}$ \\
$11 d$ e 12d & $13 \mathrm{~d}$ e 14d & $\mathrm{SO}_{2} \mathrm{C}_{6} \mathrm{H}_{5}$ \\
$11 \mathrm{e}$ & $13 \mathrm{e}$ & $\mathrm{SO}_{2} \mathrm{C}_{6} \mathrm{H}_{4} \mathrm{CH}_{3}$ \\
\hline
\end{tabular}
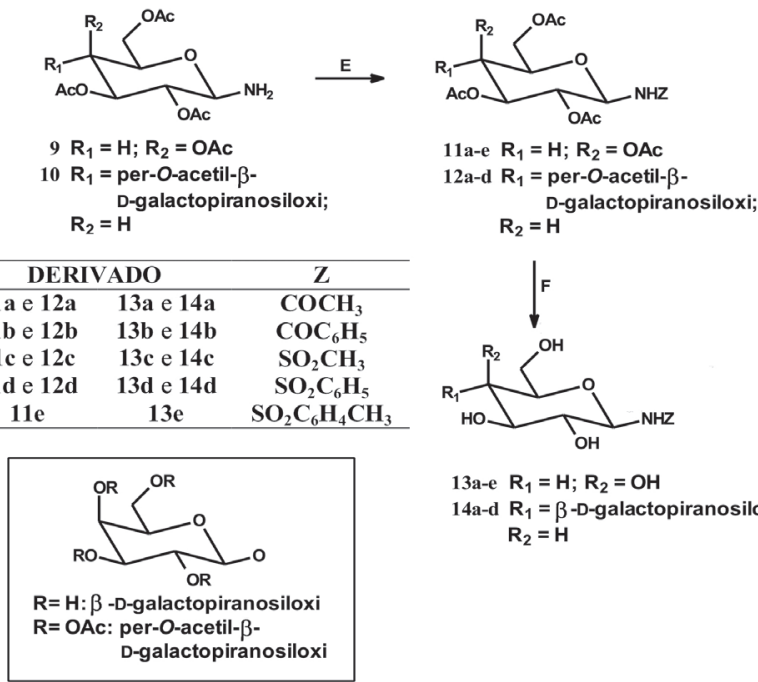

13a-e $\mathbf{R}_{1}=\mathrm{H} ; \mathbf{R}_{\mathbf{2}}=\mathrm{OH}$

14a-d $R_{1}=\beta$-D-galactopiranosiloxi; $\mathbf{R}_{2}=\mathbf{H}$ 
Tabela 1. Dados das reações de obtenção de 11a-e e 12a-d

\begin{tabular}{|c|c|c|c|c|}
\hline Glicosilamina/Reagente (relação molar) & Tempo de reação (min) & Produto desejado & Método de purificação & Rend. $^{\S}(\%)$ \\
\hline $9 /\left(\mathrm{CH}_{3} \mathrm{CO}\right)_{2} \mathrm{O}(1: 5,5)$ & 120 & 11a & - & 80 \\
\hline $9 / \mathrm{C}_{6} \mathrm{H}_{5} \mathrm{COCl}(1: 4,6)$ & 60 & 11b & - & 88 \\
\hline $9 / \mathrm{CH}_{3} \mathrm{SO}_{2} \mathrm{Cl}(1: 4,5)$ & 150 & $11 c$ & Recristalização (isopropanol) & 73 \\
\hline $9 / \mathrm{C}_{6} \mathrm{H}_{5} \mathrm{SO}_{2} \mathrm{Cl}(1: 2)$ & 80 & 11d & Recristalização (água) & 78 \\
\hline 9/4- $\mathrm{CH}_{3}-\mathrm{C}_{6} \mathrm{H}_{4} \mathrm{SO}_{2} \mathrm{Cl}(1: 2)$ & 140 & $11 \mathrm{e}$ & - & 69 \\
\hline $\mathbf{1 0} /\left(\mathrm{CH}_{3} \mathrm{CO}\right)_{2} \mathrm{O}(1: 25)$ & 180 & $12 \mathbf{a}$ & - & $97^{¥}$ \\
\hline $\mathbf{1 0} / \mathrm{C}_{6} \mathrm{H}_{5} \mathrm{COCl}(1: 4)$ & 90 & $12 b$ & Cromatografia em coluna & $60^{*}$ \\
\hline $\mathbf{1 0} / \mathrm{CH}_{3} \mathrm{SO}_{2} \mathrm{Cl}(1: 3)$ & 60 & $12 c$ & Cromatografia em coluna & 30 \\
\hline $\mathbf{1 0} / \mathrm{C}_{6} \mathrm{H}_{5} \mathrm{SO}_{2} \mathrm{Cl}(1 ; 2)$ & 60 & 12 & Cromatografia em coluna & 19 \\
\hline
\end{tabular}

$\S$ Rendimentos calculados a partir de 7e 8; ${ }^{¥}$ Mistura 12a e anômero $\alpha$ 12:1; "Mistura 12b e anômero $\alpha$ 6:1

A D-galactose (1) e a lactose (2) foram submetidas à peracetilação e os derivados peracetilados $(\mathbf{3}$ e $\mathbf{4})$ foram transformados nos brometos de glicosila correspondentes, 5 e $\mathbf{6}^{19,20}$. Em seguida foram obtidos os intermediários 7 e 8, com rendimentos de 58 e $70 \%$, respectivamente, pela substituição do bromo em $\mathrm{C}-1$ pelo grupo azido ${ }^{21,22}$. As glicosilaminas $\mathbf{9}$ e $\mathbf{1 0}$ foram preparadas a partir dos derivados 1azido-1-desoxi correspondentes (7 e 8), por reação de hidrogenação catalítica do grupo azido. Glicosilaminas são reconhecidamente instáveis, susceptíveis à anomerização e a reações de hidrólise ${ }^{17,23}$. Em vista disto, as glicosilaminas $\mathbf{9}$ e 10, logo que obtidas, foram prontamente convertidas nas amidas e sufonamidas 13a-e e 14a-d, sem purificação prévia, pela reação com os agentes acilantes e sulfonilantes adequados. Mesmo tomando-se o cuidado de se utilizar nas reações subseqüientes as glicosilaminas $\mathbf{9}$ e $\mathbf{1 0}$ logo que preparadas, eventualmente, foram observados como produtos destas reações misturas anoméricas de amidas e sulfonaminas. Da preparação de duas amidas e de uma sulfonamida, os anômeros $\beta$ 11a, 11b e 11e foram obtidos puros, conforme evidenciado pela observação dos dupletos referentes a $\mathrm{H}-1$ nos espectros de RMN de ${ }^{1} \mathrm{H}$, com constantes de acoplamento escalar $\left(J_{1,2} 9,0-9,8\right)$ características de acoplamentos entre hidrogênios antiperiplanares em anéis piranosídicos. Das outras reações foram obtidas misturas anoméricas com proporções variadas, sempre com predominância do anômero de configuração $\beta$. Utilizando-se cromatografia em coluna de sílica, foi possível obter os anômeros $\beta$ 12c e 12d e por meio de recristalização foram obtidos os anômeros $\beta$ 11c e 11b. Da reação de acilação de $\mathbf{1 0}$, que levaria à amida $\mathbf{1 2 a}$, de configuração $\beta$, foi obtida uma mistura de proporção aproximada de $\beta / \alpha$ de 12:1 (as proporções entre os anômeros foram determinadas por RMN de ${ }^{1} \mathrm{H}$ ). Mesmo sendo difícil a separação dos anômeros por cromatografia, obteve-se uma pequena quantidade de 12a pura, suficiente para sua caracterização espectroscópica. O produto bruto obtido da síntese da benzamida desejada 12b, após purificação por cromatografia em coluna de sílica, conduziu à mistura dos epímeros $\beta(\mathbf{1 2 b})$ e $\alpha$ na proporção 6/1. Na Tabela 1 estão relatadas as relações molares entre as glicosilaminas $\mathbf{9}$ e $\mathbf{1 0}$ e os reagentes acilantes ou sulfonilantes, os tempos de cada reação, assim como os procedimentos de purificação e os rendimentos dos produtos obtidos.

As amidas 12a-b, juntamente com os seus anômeros $\alpha$, e as que foram obtidas como epímeros $\beta$ puros (11a-e e 12c-d) foram submetidas à reação de transterificação com metóxido de sódio em metanol ${ }^{24}$. As amidas e sulfonamidas desprotegidas resultantes 13ae e 14a-d, obtidas com rendimentos de $49-98 \%$, foram purificadas, quando necessário, por recristalização (Tabela 2).

Com a finalidade de se avaliar se as amidas e sulfonamidas seriam susceptíveis à anomerização durante o processo de sua obtenção, a sulfonamida 12c pura foi colocada nas condições empregadas para sua preparação. A formação do anômero $\alpha$ não foi observada enquanto a mistura reagente foi deixada em banho de gelo,
Tabela 2. Tempo de reação e solventes empregados na recristalizações de 13a-e e 14a-d

\begin{tabular}{lccc}
\hline Produto & $\begin{array}{c}\text { Tempo de } \\
\text { reação }(\mathrm{min})\end{array}$ & $\begin{array}{c}\text { Solvente de } \\
\text { recristalização }\end{array}$ & $\begin{array}{c}\text { Rend. } \\
(\%)\end{array}$ \\
\hline 13a & 30 & - & 98 \\
13b & 60 & - & 95 \\
13c & 60 & - & 87 \\
13d & 60 & isopropanol & 92 \\
13e & 90 & - & 91 \\
14a & 60 & isopropanol/água 4:1 & 60 \\
14b & 60 & metanol & 78 \\
14c & 60 & metanol & 49 \\
14d & 90 & metanol/água $9: 1$ & 53 \\
\hline
\end{tabular}

nem quando aquecida até $60{ }^{\circ} \mathrm{C}$ por $24 \mathrm{~h}$. Isto evidenciou que as amidas e sulfonamidas de configuração $\alpha$ foram, possivelmente, formadas a partir da glicosilamina de mesma configuração, resultante da anomerização parcial das glicosilaminas 9 e 10, o que pode ter ocorrido na reação de sua formação ou durante as reações de acilação e sulfonilação.

\section{Ensaio de inibição de hemaglutinação}

A afinidade das amidas e sulfonamidas 13a-e e 14a-d por LEC e RCA120 foi avaliada em ensaio de inibição de hemaglutinação mediado por estas lectinas ${ }^{25}$. As potências relativas à inibição promovida pela D-galactose (1), para cada um dos derivados e para lactose (2), estão mostradas na Tabela 3.

Tabela 3. Potência relativa dos derivados 13a-e e 14a-d quanto à inibição da hemaglutinação mediada por LEC e RCA120, em relação à D-galactose $(\mathbf{1})$

\begin{tabular}{lcc}
\hline Substância & \multicolumn{2}{c}{ Potência Relativa } \\
& LEC & RCA120 \\
\hline $\mathbf{1}$ & 1 & 1 \\
$\mathbf{2}$ & 4 & 6 \\
$\mathbf{1 3 a}^{*}$ & 0,25 & 0,5 \\
$\mathbf{1 3 b}^{*}$ & 0,25 & 1 \\
$\mathbf{1 3 c}^{*}$ & 0,25 & 0,25 \\
$\mathbf{1 3 d}^{*}$ & 0,5 & 0,5 \\
$\mathbf{1 3 e}^{*}$ & 0,5 & 0,5 \\
$\mathbf{1 4 a}^{\#}$ & 3 & 4 \\
$\mathbf{1 4 b}^{\#}$ & 4 & 4 \\
$\mathbf{1 4 c}^{\#}$ & 4 & 4 \\
$\mathbf{1 4 d}^{\#}$ & 4 & 2 \\
\hline
\end{tabular}

*derivados de D-galactose; \#derivados de lactose 
As modificações efetuadas em $\mathbf{1}$ e $\mathbf{2}$ não contribuíram para aumentar a afinidade de 13a-e e 14a-d por LEC e RCA120. As amidas 14a e 14b e as sulfonamidas $14 \mathrm{c}$ e $14 \mathrm{~d}$, derivadas da lactose (2), apresentaram, praticamente, a mesma afinidade que esta em relação à LEC, e foram, de forma geral, um pouco menos eficientes em relação à RCA120. Já as amidas 13a e 13b e as sulfonamidas 13c-e, derivadas da D-galactose (1), apresentaram, à exceção de 13b, afinidade nitidamente inferior a esta, em relação às duas lectinas. A afinidade da lactose (2), quatro vezes maior pela LEC e seis vezes pela RCA120, em relação à do monossacarídeo D-galactose, pode ser explicada pelas possibilidades de interações das lectinas com grupos hidroxila do resíduo de glicose, mediadas por moléculas de água ${ }^{26,27}$. A introdução dos grupos amido e sulfonamido na posição anomérica não promoveu o aumento das interações com as lectinas. Especula-se que a diminuição da afinidade por ambas lectinas tenha sido decorrente de uma rede de solvatação mais eficiente do ligante, favorecida por ligações de hidrogênio entre as moléculas de água e os grupos amido e sulfonamido destes derivados, o que levaria a um aumento da energia de dessolvatação e conseqüente diminuição da interação desses carboidratos com as lectinas ${ }^{28}$.

\section{CONCLUSÕES}

Foram sintetizados nove derivados de carboidratos, os quais foram avaliados quanto as suas interações com as lectinas LEC e RCA120, em ensaio de inibição de hemaglutinação. A substituição das hidroxilas anoméricas por grupos amido e sulfonamido não resultou em aumento da afinidade destes compostos pelas lectinas estudadas.

\section{PARTE EXPERIMENTAL}

\section{Procedimentos gerais}

As temperaturas de fusão foram determinadas em aparelho Microquímica MQAs 301 e não foram corrigidas. Os valores de poder rotatório específico, $[\alpha]_{D}$, foram medidos em polarímetros ADP220 Bellinghan + Stanley Ltd. ou Perkin Elmer 341, a $20{ }^{\circ} \mathrm{C}$. Os espectros de $\mathrm{RMN}$ de ${ }^{1} \mathrm{H}$ e de ${ }^{13} \mathrm{C}$ foram registrados em espectrômetros Bruker Avance DPX-200 ou DRX-400. Como referência interna foi utilizado o tetrametilsilano. Os espectros no IV foram registrados em aparelho Spectrum One, Perkin-Elmer. A evolução das reações foi acompanhada por cromatografia em camada delgada de sílica, utilizando-se como revelador solução de ácido sulfúrico $15 \% \mathrm{v} / \mathrm{v}$ em etanol e aquecimento da placa em estufa. As purificações por cromatografia em coluna de sílica foram realizadas com sílica gel 60, 70-230 mesh (Merck). As lectinas vegetais (LEC e RCA120) empregadas nos ensaios de hemaglutinação foram isoladas e purificadas segundo técnicas descritas na literatura $^{29}$. Para as atribuições dos sinais nos espectros de RMN, adotou-se a seguinte convenção: para os derivados da D-galactose, os átomos do açúcar foram numerados de 1 a 6 . Para os derivados da lactose, o resíduo de D-glicose foi numerado de 1 a 6 e o de Dgalactose, de 1' a 6'. Os substituintes aromáticos em C-1, foram numerados de 7 a 10 (carbono ligado aos grupos carbonila/sulfonila, posições orto, meta e para, respetivamente).

Os compostos 3-6 foram preparados conforme procedimentos descritos na literatura ${ }^{21}$.

\section{2,3,4,6-tetra- $O$-acetil-1-azido-1-desoxi- $\beta$-D-galactopiranose (7)}

Em um balão de fundo redondo de $250 \mathrm{~mL}$ foram adicionados $14 \mathrm{~g}(33,98 \mathrm{mmol})$ de 5 e $80 \mathrm{~mL}$ de acetona, seguido de 2,86 g (44,00 mmol) de azida de sódio dissolvidos em $40 \mathrm{~mL}$ de água destilada. Deixou-se a mistura sob agitação magnética, à temperatura ambiente, por $6 \mathrm{~h}$. Após o término da reação, a mistura reagente foi vertida em cápsula de porcelana e a acetona evaporada sob ventilação. O resíduo aquoso obtido foi extraído com diclorometano ( 3 x $30 \mathrm{~mL})$ e a fase orgânica foi lavada com água destilada (3 x 30 $\mathrm{mL}$ ). A fase orgânica foi secada com sulfato de sódio anidro e o solvente foi destilado em evaporador rotatório, fornecendo um óleo que foi pré-purificado por filtração em coluna de sílica com acetato de etila. $\mathrm{O}$ filtrado foi concentrado em evaporador rotatório e o sólido obtido foi recristalizado com álcool isopropílico. Foram obtidos 8,4 g (22,5 mmol; 66\%) de 7. Sólido branco cristalino. Tf: 91,5-93,0 ${ }^{\circ} \mathrm{C}$ (lit.: $\left.91-92{ }^{\circ} \mathrm{C}^{30}\right)$. $[\alpha]_{\mathrm{D}}-4,8\left(c 1, \mathrm{CHCl}_{3}\right)\left(\right.$ lit.: $[\alpha]_{\mathrm{D}}$ $\left.8,0, c 1, \mathrm{CHCl}_{3}{ }^{31}\right)$. IV $\left(v_{\text {máx }}, \mathrm{cm}^{-1}\right): 2126,1737,1211,1054$.

\section{2,3,6-tri- $O$-acetil-4- $O$-(2,3,4,6-tetra- $O$-acetil- $\beta$-D- galactopiranosil)-1-azido-1-desoxi- $\beta$-D-glicopiranose (8)}

Em um balão de fundo redondo de $250 \mathrm{~mL}$ foram dissolvidos $10 \mathrm{~g}(14,32 \mathrm{mmol})$ de $\mathbf{6} \mathrm{em} 70 \mathrm{~mL}$ de acetona. Foi adicionada uma solução de $4 \mathrm{~g}(61,53 \mathrm{mmol})$ de azida de sódio em $40 \mathrm{~mL}$ de água destilada. O balão foi acoplado a um condensador de refluxo e a solução reagente foi deixada sob agitação magnética, a $45^{\circ} \mathrm{C}$, por 3 h. Após o término da reação, a solução reagente foi vertida em uma cápsula de porcelana e a acetona foi evaporada sob ventilação. O resíduo aquoso foi extraído com diclorometano (3 x $25 \mathrm{~mL})$. As camadas orgânicas foram reunidas, extraídas com água destilada $(2 \times 30 \mathrm{~mL})$. A fase orgânica foi secada com sulfato de sódio anidro e o solvente foi destilado em evaporador rotatório. Foi obtido um óleo transparente que quando triturado com éter de petróleo solidificou. O sólido branco foi purificado por cromatografia em coluna de sílica gel, utilizando como eluente uma mistura de hexano/ acetato de etila $(1: 1 \mathrm{v} / \mathrm{v})$. Foram obtidos $6,6 \mathrm{~g}(9,97 \mathrm{mmol} ; 70 \%)$ de 8. Sólido branco amorfo. Tf: $62,8-63,7{ }^{\circ} \mathrm{C}$ (lit.: $69-70{ }^{\circ} \mathrm{C}^{32}$ ). $[\alpha]_{\mathrm{D}}-91,6\left(c \mathrm{0}, 52, \mathrm{CHCl}_{3}\right)$. (lit.: $[\alpha]_{\mathrm{D}}-26,8, c 1,13, \mathrm{CHCl}_{3}{ }^{33} ;[\alpha]_{\mathrm{D}}$ $\left.18 ; c 0,64, \mathrm{CHCl}_{3}^{34}\right)$. IV $\left(\mathrm{v}_{\text {máx }}, \mathrm{cm}^{-1}\right): 2119,1740,1211,1043$.

$2,3,4,6$-tetra- $O$-acetil- $\beta$-D-galactopiranosilamina (9) e 2,3,6-tri$O$-acetil-4- $O$-(2,3,4,6-tetra- $O$-acetil- $\beta$-D-galactopiranosil)- $\beta$-Dglicopiranosilamina $(10)^{32,35}$

Em um balão de fundo redondo de $50 \mathrm{~mL}$ foram colocados 50 mg de paládio-carvão $10 \%, 5 \mathrm{~mL}$ de metanol anidro e $1 \mathrm{~g}$ de $7 \mathrm{ou}$ 8 (2,67 e 1,51 mmol, respectivamente) dissolvido em $10 \mathrm{~mL}$ de metanol anidro. O balão foi fechado com septos de borracha, eliminou-se o ar passando-se nitrogênio gasoso e a mistura deixada sob agitação magnética, à temperatura ambiente e sob atmosfera de gás hidrogênio. Após o término da reação, o catalisador foi removido por filtração e a solução recolhida foi concentrada em evaporador rotatório a $40{ }^{\circ} \mathrm{C}$. As glicosilaminas foram obtidas como sólidos brancos e utilizadas nas reações subseqüentes, imediatamente, sem purificação prévia.

\section{Síntese das amidas e sulfonamidas}

\section{Sintese de 11a-e e 12a-d: procedimento geral}

Em balão de fundo redondo, imerso em banho de gelo, a glicosilamina 9 ou 10 foi dissolvida em $5 \mathrm{~mL}$ de piridina. $\mathrm{O}$ agente acilante ou sulfonilante foi adicionado gota a gota, a mistura reagente foi agitada à temperatura ambiente por 60-180 min. Após o término da reação, a solução reagente foi vertida em gelo pilado e submetida à extração com diclorometano $(3$ x $15 \mathrm{~mL})$. As camadas orgânicas reunidas foram lavadas com solução de ácido clorídrico $1 \mathrm{~mol} / \mathrm{L}$ (5 x $10 \mathrm{~mL}$ ) e, em seguida, com água destilada até a 
fase aquosa da última extração apresentar $\mathrm{pH}$ igual a 6. A fase orgânica foi secada com sulfato de sódio anidro e o solvente foi destilado em evaporador rotatório. As relações molares de glicosilaminas e de reagentes acilantes ou sulfonilantes, os tempos de reação, os procedimentos de purificação e os rendimentos dos produtos encontram-se relatados na Tabela 1.

\section{2,3,4,6-tetra-O-acetil-1-acetilamino-1-desoxi- $\beta$ - $D$ - galactopiranose (11a)}

Foram obtidos $0,83 \mathrm{~g}$ de 11a $(2,14 \mathrm{mmol} ; 80 \%$ a partir de 7$)$. Sólido branco. Tf: $169,6-170,8^{\circ} \mathrm{C}$ (lit.: $\left.173{ }^{\circ} \mathrm{C}^{36}\right)$. $[\alpha]_{\mathrm{D}}+33,0(c 2$, $\left.\mathrm{CHCl}_{3}\right)$ (lit.: $[\alpha]_{\mathrm{D}}+34,7\left(c 2, \mathrm{CHCl}_{3}{ }^{36}\right)$. IV $\left(\nu_{\text {máx }}, \mathrm{cm}^{-1}\right): 3200,1756$, $1737,1666,1223,1050 . \mathrm{RMN}$ de ${ }^{1} \mathrm{H}\left(\delta ; \mathrm{CDCl}_{3} ; 200 \mathrm{MHz}\right): 6,50$ $\left(\mathrm{d} ; J_{\mathrm{NH}, 1} 8,9 \mathrm{~Hz} ; \mathrm{N} \underline{\mathrm{H}}\right) ; 5,45(\mathrm{~d} ; 1 \mathrm{H} ; J 1,7 \mathrm{~Hz}) ; 5,30-5,05(\mathrm{~m} ; 3 \mathrm{H})$; 4,17-4,02 (m;3H); 2,15 (s; NHCOC $\left.\underline{H}_{3}\right) ; 2,07\left(\mathrm{~s} ; \mathrm{OCOC}_{3}\right) ; 2,15$ $\left(\mathrm{s} ; \mathrm{OCOCH}_{3}\right) ; 2,04\left(\mathrm{~s} ; \mathrm{OCOC}_{3}\right) ; 1,99\left(\mathrm{~s} ; \mathrm{OCOC}_{3}\right) ; \mathrm{RMN}$ de ${ }^{13} \mathrm{C}$ $\left(\delta ; \mathrm{CDCl}_{3} ; 50 \mathrm{MHz}\right): 171,26,170,45,170,09,169,80$ (4C; $\left.\mathrm{OCOCH}_{3}\right) ; 169,8\left(\mathrm{NHCOCH}_{3}\right) ; 78,57$ (C-1); 72,50 (C-3); 70,99 (C5); 68,44 (C-2); 67,34 (C-4); 61,32 (C-6); 23,37 (NHCOCH $\left.)_{3}\right)$ 20,81, 20,75, 20,65, 20,59 (4C; $\left.\mathrm{OCO}_{\mathbf{H}}\right)_{3}$.

\section{2,3,4,6-tetra-O-acetil-1-benzoilamino-1-desoxi- $\beta$ - $D$ - galactopiranose $(\mathbf{1 1 b})$}

Foram obtidos $1,06 \mathrm{~g}$ de $\mathbf{1 1 b}(2,35 \mathrm{mmol} ; 88 \%$ a partir de 7$)$. Sólido branco. Tf: $142,5-144,5{ }^{\circ} \mathrm{C}$ (lit.: $\left.148-149{ }^{\circ} \mathrm{C}^{37}\right)$. $[\alpha]_{\mathrm{D}}+6,0(c$ 2, $\left.\mathrm{CHCl}_{3}\right)\left(\right.$ lit.: $\left.[\alpha]_{\mathrm{D}}+4,9, c 1, \mathrm{CHCl}_{3}^{37}\right)$. IV $\left(\mathrm{v}_{\text {máx }}, \mathrm{cm}^{-1}\right)$ : 3324, 1736, $1677,1605,1212,1055$. RMN de ${ }^{1} \mathrm{H}\left(\delta ; \mathrm{CDCl}_{3} ; 200 \mathrm{MHz}\right): 7,78(\mathrm{~d} ;$ $J_{8.9} 7 \mathrm{~Hz}$; H-8); 7,58-7,41 (m; H-9 e H-10); 7,12 (d; $J_{\mathrm{NH}, 1} 8,89 \mathrm{~Hz}$; $\mathrm{N} \underline{\mathrm{H}}) ; 5,49-5,23(\mathrm{~m} ; 4 \mathrm{H}) ; 4,18-4,09(\mathrm{~m} ; 3 \mathrm{H}) ; 2,15\left(\mathrm{~s} ; \mathrm{OCOC}_{3}\right) ; 2,11$ $\left(\mathrm{s} ; \mathrm{OCOC}_{3}\right) ; 2,04\left(\mathrm{~s} ; \mathrm{OCOC}_{3}\right) ; 2,02\left(\mathrm{~s} ; \mathrm{OCOC}_{3}\right) . \mathrm{RMN}$ de ${ }^{13} \mathrm{C}$ $\left(\delta ; \mathrm{CDCl}_{3} ; 50 \mathrm{MHz}\right): 171,90,170,50,170,17,169,91\left(4 \mathrm{C} ; \mathrm{OCOCH}_{3}\right)$; 167,19 (NHCO); 132,99 (C-7); 132,50 (C-10); 128,87 (C-8); 127,36 (C-9); 79,32 (C-1); 72,48 (C-3); 72,48 (C-5); 70,93 (C-2); 68,69 (C-

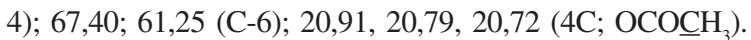

\section{2,3,4,6-tetra-O-acetil-1-desoxi-1-metanossulfonilamino- $\beta-D-$ galactopiranose $(\mathbf{1 1 c})$}

O produto bruto da reação foi recristalizado com isopropanol. Foram obtidos $0,83 \mathrm{~g}$ de 11c $(1,95 \mathrm{mmol}$; $73 \%$ a partir de 7$)$. Sólido branco. Tf: $187-188^{\circ} \mathrm{C}$. $[\alpha]_{\mathrm{D}}+31,8\left(c 1, \mathrm{CHCl}_{3}\right)$. IV $\left(v_{\text {máx }}, \mathrm{cm}^{-1}\right)$ : $3188,1754,1738,1713,1333,1215,1152,1035$. RMN de ${ }^{1} \mathrm{H}(\delta$; $\left.\mathrm{CDCl}_{3} ; 200 \mathrm{MHz}\right): 8,46\left(\mathrm{~d} ; J_{\mathrm{NH}}, 9,9 \mathrm{~Hz} ; \mathrm{NH}\right) ; 5,26(\mathrm{~m} ; 2 \mathrm{H}) ; 5,0(\mathrm{t}$; $\left.J_{1, \mathrm{NH}}=9,8 \mathrm{~Hz} ; \mathrm{H}-1\right) ; 4,85 ;\left(\mathrm{t} ; J_{2.1} 8,9 \mathrm{~Hz} ; \mathrm{H}-2\right) ; 4,32-4,26$ (m; H-3); 4,11-4,04 (m;2H);2,95 (s; $\left.\mathrm{SO}_{2} \underline{\mathrm{H}}_{3}\right) ; 2,10\left(\mathrm{~s} ; \mathrm{OCOC}_{3}\right) ; 2,02(\mathrm{~s} ;$ $\left.\mathrm{OCOC}_{3}\right) ; 1,98\left(\mathrm{~s} ; \mathrm{OCOC}_{3}\right) ; 1,91\left(\mathrm{~s} ; \mathrm{OCOC}_{3}\right) . \mathrm{RMN}$ de ${ }^{13} \mathrm{C}(\delta ;$ $\left.\mathrm{CDCl}_{3} ; 50 \mathrm{MHz}\right): 169,91,169,43,169,17\left(4 \mathrm{C} ; \mathrm{OCOCH}_{3}\right) ; 82,23$ (C-1); 71,23 (C-3); 70,83 (C-5); 67,42 (C-2 e C-4); 61,49 (C-6); $\left.42,82\left(\mathrm{SO}_{2} \underline{\mathrm{CH}}\right)_{3}\right) ; 20,53,20,48,20,35\left(4 \mathrm{C} ; \mathrm{OCOCH}_{3}\right)$.

\section{2,3,4,6-tetra-O-acetil-1-benzenossulfonilamino-1-desoxi- $\beta$ - $D$ - galactopiranose $(11 \mathrm{~d})$}

$\mathrm{O}$ produto bruto da reação foi recristalizado com água. Foram obtidos 1,01 g de 11d (2,08 mmol; 78\% a partir de 7). Sólido branco. Tf: $112,5-114,0^{\circ} \mathrm{C} .[\alpha]_{\mathrm{D}}+23,9\left(c 1, \mathrm{CHCl}_{3}\right)$. IV $\left(v_{\text {máx }}, \mathrm{cm}^{-1}\right)$ : $3133,1751,1717,1485,1447,1331,1159,1210,1046$. RMN de ${ }^{1} \mathrm{H}\left(\delta ; \mathrm{CDCl}_{3} ; 200 \mathrm{MHz}\right): 7,89\left(\mathrm{~d} ; J_{8.9} 6,8 \mathrm{~Hz} ; \mathrm{H}-8\right) ; 7,58-7,46(\mathrm{~m} ;$ H-9 e H-10); 5,81 (d; $\left.J_{\mathrm{NH}, 1}=9,7 \mathrm{~Hz} ; \underline{\mathrm{H}}\right) ; 5,38$ (d; $\left.J 1,9 \mathrm{~Hz} ; \mathrm{H}-4\right)$; 5,09-5,00 (m; 2H); 4,87-4,77 (m; 1H); 4,01-3,80 (m;3H); 2,09 (s; $\left.\mathrm{OCOC}_{3}\right) ; 2,01\left(\mathrm{~s} ; \mathrm{OCOC}_{3}\right) ; 1,97\left(\mathrm{~s} ; \mathrm{OCOCH}_{3}\right) . \mathrm{RMN} \mathrm{de}{ }^{13} \mathrm{C}(\delta$; $\left.\mathrm{CDCl}_{3} ; 50 \mathrm{MHz}\right): 171,08,170,44,170,12,169,92\left(4 \mathrm{C} ; \mathrm{OCOCH}_{3}\right)$; 141,50 (C-7); 133,02 (C-10); 129,06 (C-8); 127,18 (C-9); 83,32 (C-1); 72,30 (C-3); 70,89 (C-5); 67,80 (C-2); 67,02 (C-4); 61,05 (C-6); 20,77, 20,72, 20,67, 20,62 (4C; $\left.\mathrm{OCOC}_{3}\right)$.
2,3,4,6-tetra-O-acetil-1-desoxi-1-(4-metilbenzenossulfonilamino)$\beta$-D-galactopiranose (11e)

Foram obtidos $0,92 \mathrm{~g}$ de $11 \mathrm{e}(1,84 \mathrm{mmol}$; $69 \%$ a partir de 7$)$. Sólido branco. Tf: $153,8-154,7{ }^{\circ} \mathrm{C}$. $[\alpha]_{\mathrm{D}}+11,9$ (c 1, $\left.\mathrm{CHCl}_{3}\right)$. IV $\left(v_{\text {máx }}, \mathrm{cm}^{-1}\right): 3185,1751,1720,1595,1480,1434,1341,1213,1047$. RMN de ${ }^{1} \mathrm{H}\left(\delta ; \mathrm{CDCl}_{3} ; 200 \mathrm{MHz}\right): 7,76\left(\mathrm{~d} ; J_{8.9} 8,2 \mathrm{~Hz} ; \mathrm{H}-8\right) ; 7,28$ (d; $\left.J_{9,8} 8 \mathrm{~Hz} ; \mathrm{H}-9\right) ; 5,78$ (d; $\left.J_{\mathrm{NH}, 1} 9,8 \mathrm{~Hz} ; \mathrm{NH}\right) ; 5,38$ (sl; H-4); 5,085,05 (m; 2H); 4,81 (t; 1H; J 8,46 Hz); 3,99-3,84 (m; H-5; H-6a e H6b); 2,41 (s; $\left.\operatorname{ArC}_{3}\right) ; 2,09$ (s; OCOC $\left.\underline{H}_{3}\right) ; 2,02\left(\mathrm{~s} ; \mathrm{OCOC}_{3}\right) ; 1,97$ (s; $\left.\mathrm{OCOC}_{3}\right)$. RMN de ${ }^{13} \mathrm{C}\left(\delta ; \mathrm{CDCl}_{3} ; 50 \mathrm{MHz}\right): 171,05,170,44$, 170,14, 169,94 (4C; $\left.\mathrm{OCOCH}_{3}\right) ; 143,85$ (C-7); 138,55 (C-10); 129,60 (C-8); 127,23 (C-9); 83,29 (C-1); 72,23 (C3); 70,9 (C-5); 67,78 (C-2); 67,06 (C-4); 61,04 (C-6); 21,64, 20,73, 20,67 (5C; ArCh $_{3}$ e $\left.\mathrm{OCOCH}_{3}\right)$.

2,3,6-tri-O-acetil-1-acetilamino-4-O-(2,3,4,6-tetra-O-acetil- $\beta$ - $D$ galactopiranosil)-1-desoxi- $\beta$-D-glicopiranose (12a)

A acetamida 12a (anômero $\beta$ ) foi obtida em mistura com seu isômero $\alpha$, na proporção de 12:1 $(0,99 \mathrm{~g} ; 1,47 \mathrm{mmol}$; $97 \%$ a partir de 8). Esta mistura foi utilizada na reação de transterificação seguinte, sem purificação prévia. Para fins de caracterização, foram purificados $400 \mathrm{mg}$ da mistura de anômeros por cromatografia em coluna de sílica gel (eluição em gradiente hexano/acetato de etila). Foram obtidos $124 \mathrm{mg}(0,18 \mathrm{mmol})$ de 12a puro. Sólido branco amorfo. Tf: 100,1-102,1. (lit.: $\left.131-132{ }^{\circ} \mathrm{C}^{38}\right)$. $[\alpha]_{\mathrm{D}}+11,9\left(c 1, \mathrm{CHCl}_{3}\right)\left(\right.$ lit.: $[\alpha]_{\mathrm{D}}$ $\left.+11,7, c 1, \mathrm{CH}_{2} \mathrm{Cl}_{2}^{38}\right)$. IV $\left(v_{\text {máx }}, \mathrm{cm}^{-1}\right): 3323,1744,1681,1214,1038$. RMN de ${ }^{1} \mathrm{H}\left(\delta ; \mathrm{CDCl}_{3} ; 400 \mathrm{MHz}\right): 6,26\left(\mathrm{~d} ; J_{\mathrm{NH}, 1} 9,3 \mathrm{~Hz} ; \mathrm{NH}\right) ; 5,35$ (dd; $J_{4^{\prime}, 5}, 0,9 \mathrm{~Hz} ; J_{4,3}, 3,4 \mathrm{~Hz} ; \mathrm{H}-4$ '); 5,29 (t; $J_{3,2}=J_{3,4} 9,3 \mathrm{~Hz} ; \mathrm{H}-3$ ); $5,20\left(\mathrm{t} ; J_{1, \mathrm{NH}}=J_{1,2} 9,3 \mathrm{~Hz} ; \mathrm{H}-1\right) ; 5,10\left(\mathrm{dd} ; J_{2,3}, 10,4 \mathrm{~Hz} ; J_{2,1}, 7,8 \mathrm{~Hz} ; \mathrm{H}-\right.$ 2'); 4,95 (dd; $\left.J_{3,4}, 3,4 \mathrm{~Hz} ; J_{3,2}, 10,4 \mathrm{~Hz} ; \stackrel{\mathrm{H}-3}{\prime}\right)$ ); 4,82 (t; $J_{2,1}=J_{2,3} 9,3$ Hz; H-2); 4,47 (d; $\left.J_{1,2}, 7,8 \mathrm{~Hz} ; \mathrm{H}^{\prime} 1^{\prime}\right) ; 4,43$ (dd; $J_{6 \mathrm{a}, 5} 1,3 \mathrm{~Hz} ; J_{6 \mathrm{a}, 6 \mathrm{~b}} 12,1$ Hz; H-6a); 4,17-4,12 (m; 2H; H-6b e H-6a'); 4,07 (dd; $J_{6 b}, 5,7,3$ Hz; $J_{6 b^{\prime}, 6 a^{\prime}}, 11,1 \mathrm{~Hz} ; \mathrm{H}-6 \mathrm{~b}$ '); 3,87 (tl; $J_{5^{\prime}, 6}=J_{5^{\prime}, 4}, 7,3 \mathrm{~Hz} ; \mathrm{H}-5$ '); 3,79-3,72 (m; $2 \mathrm{H}, \mathrm{H}-4$ e H-5); 2,15 (s; OCOC $\left.\underline{\mathrm{H}}_{3}\right) ; 2,11$ (s; OCOC $\left.\underline{\mathrm{H}}_{3}\right) ; 2,07$ (s; $\left.\mathrm{OCOC}_{3}\right) ; 2,05\left(\mathrm{~s} ; \mathrm{OCOC}_{3}\right) ; 2,04\left(\mathrm{~s} ; \mathrm{OCOC}_{3}\right) ; 1,98\left(\mathrm{~s} ; \mathrm{OCOC} \underline{\mathrm{H}}_{3}\right)$; $1,96\left(\mathrm{~s} ; \mathrm{OCOC}_{3}\right)$. RMN de ${ }^{13} \mathrm{C}\left(\delta ; \mathrm{CDCl}_{3} ; 100 \mathrm{MHz}\right): 171,32$ $\left(\mathrm{NHCOCH}_{3}\right)$; 170,37, 170,30, 170,16, 170,09, 169,34, 169,00 (7C; $\left.\mathrm{OCOCH}_{3}\right) ; 100,89$ (C-1'); 78,09 (C-1); 75,97 (C-4); 74,50 (C-5); 72,42 (C-3); 71,02; 71,00 (C-2 e C-3'); 70,76 (C-5'); 69,05 (C-2'); 66,68 (C-4'); 62,03 (C-6); 60,89 (C-6'); 23,36 (C-7); 20,86, 20,77, 20,69, 20,63, 20,60, 20,50 (7C; $\left.\mathrm{OCO}^{\mathrm{CH}}{ }_{3}\right)$.

\section{2,3,6-tri-O-acetil-4-O-(2,3,4,6-tetra-O-acetil- $\beta$-D-galactopiranosil)-} $\underline{1 \text {-benzoilamino-1-desoxi- } \beta \text { - } D \text {-glicopiranose (12b) }}$

O material bruto obtido da síntese da benzamida 12b $(0,90 \mathrm{~g})$ foi submetido à cromatografia em coluna de sílica (hexano/acetato de etila 4:6). A partir de $300 \mathrm{mg}$ do produto bruto, foram obtidos $224 \mathrm{mg}(0,30 \mathrm{mmol} ; 60 \%$ a partir de $\mathbf{8})$ de um sólido branco caracterizado como uma mistura do anômero $\beta$ (12b) e seu isômero $\alpha$ na proporção de 6:1. Foi possível fazer a atribuição dos sinais de hidrogênio e de carbono-13 do produto predominante na mistura 12b. RMN de ${ }^{1} \mathrm{H}\left(\delta ; \mathrm{CDCl}_{3} ; 400 \mathrm{MHz}\right): 7,74\left(\mathrm{~d} ; J_{8,9} 7,4 \mathrm{~Hz} ; \mathrm{H}-8\right.$ e H-8'); 7,55-7,49 (m; H-10); 7,44 (t; $J_{9,8}=J_{9,10} 7,4$ Hz; H-9 e H-9'); 6,98 (d; $\left.J_{\mathrm{NH}, 1} 9,0 \mathrm{~Hz} ; \mathrm{NH}\right) ; 5,45-5,36$ (m; H-1, H-3 e H-4'); 5,12 (dd; $J_{2,1}, 7,8 \mathrm{~Hz} ; J_{2,3}, 10,4 \mathrm{~Hz} ; \mathrm{H}-2$ '); 4,99-4,94 (m; H-2 e H-3'); 4,48 (d; $\left.J_{1,2}, 7,8 \mathrm{~Hz} ; \mathrm{H}^{\prime}{ }^{\prime}\right)$ ); 4,46 (d; $\left.J_{6 \mathrm{a}, 6 \mathrm{~b}} 12,4 \mathrm{~Hz} ; \mathrm{H}-6 \mathrm{a}\right) ; 4,19-4,13$ (m; H-6b e H-6a'); 4,09 (dd; $J_{6 b^{\prime}, 5}, 7,1 \mathrm{~Hz} ; J_{6 b^{\prime}, 6 a^{\prime}} 11,1 \mathrm{~Hz}$; H-6b'); $3,89\left(\mathrm{t} ; J_{5,6}=J_{5,4}, 7,1 \mathrm{~Hz} ; \mathrm{H}-5\right.$ '); 3,84-3,80 (m; H-4 e H-5); 2,17 (s; $\left.\mathrm{OCOCH}_{3}\right) ; 2,16\left(\mathrm{~s} ; \mathrm{OCOC}_{3}\right) ; 2,15\left(\mathrm{~s} ; \mathrm{OCOC}_{3}\right) ; 2,11(\mathrm{~s} ;$ OCOC $\left.\underline{H}_{3}\right) ; 2,10\left(\mathrm{~s} ; \operatorname{OCOC} \underline{H}_{3}\right) ; 2,09\left(\mathrm{~s} ; \operatorname{OCOC}_{3}\right) ; 2,08(\mathrm{~s} ;$ OCOC $\left.\underline{H}_{3}\right) ; 2,07\left(\mathrm{~s} ; \operatorname{OCOC}_{3}\right) ; 2,05\left(\mathrm{~s} ; \mathrm{OCOC}_{3}\right) ; 2,03(\mathrm{~s} ;$ $\left.\mathrm{OCOC}_{3}\right) ; 2,02\left(\mathrm{~s} ; \mathrm{OCOC}_{3}\right) . \mathrm{RMN} \mathrm{de}{ }^{13} \mathrm{C}\left(\delta ; \mathrm{CDCl}_{3} ; 100 \mathrm{MHz}\right)$ : $171,75\left(\mathrm{NHCOC}_{6} \mathrm{H}_{5}\right) ; 170,34,170,15,170,07,169,32,168,99$, 
167,04 (7C; $\left.\mathrm{OCOCH}_{3}\right) ; 132,89$ (C-7); 132,37 (C-10); 128,75 (C8); 127,21 (C-9); 101,30 (C-1'); 78,79 (C-1); 76,04 (C-4); 74,55 (C-5); 72,25 (C-3); 71,20; 71,04 (C-2 e C-3'); 70,94 (C-5'); 69,06 (C-2'); 66,69 (C-4'); 62,0 (C-6); 60,93 (C-6'); 20,86, 20,80, 20,74, 20,64, 20,62, 20,50 (7C; $\left.\mathrm{OCO}_{3}\right)_{3}$.

\section{2,3,6-tri-O-acetil-4-O-(2,3,4,6-tetra-O-acetil- $\beta$ - $\underline{-}$ -}

galactopiranosil)-1-desoxi-1-metanossulfonilamino- $\beta-D-$ glicopiranose (12c)

O material bruto obtido da síntese da sulfonamida 12c foi purificado por cromatografia em coluna de sílica (hexano/acetato de etila 4:6). A partir de $890 \mathrm{mg}$ do material bruto, foram obtidos $337 \mathrm{mg}$ do anômero $\beta$ 12c $(0,42 \mathrm{mmol} ; 30 \%$ a partir de 8). Sólido branco. Tf: 99,1-103,2 ${ }^{\circ} \mathrm{C} .[\alpha]_{\mathrm{D}}+11,9\left(c 2, \mathrm{CHCl}_{3}\right)$. IV $\left(v_{\text {máx }}, \mathrm{cm}^{-1}\right): 1740,1368$, $1333,1212,1038$. RMN de ${ }^{1} \mathrm{H}\left(\delta ; \mathrm{CDCl}_{3} ; 400 \mathrm{MHz}\right): 5,53$ (d; $J_{\mathrm{NH}, 1}$ 9,1 Hz; N- $)$ ); 5,35 (d; $J_{4,3}, 3,2 \mathrm{~Hz} ; \mathrm{H}-4$ '); 5,28 (t; $J_{3,2}=J_{3,4} 9,1 \mathrm{~Hz} ; \mathrm{H}-$ 3); 5,11 (dd ; $\left.J_{2,1}, 7,9 \mathrm{~Hz} ; J_{2,3}, 10,4 \mathrm{~Hz} ; \mathrm{H}-2^{\prime}\right) ; 4,98$ (dd; $J_{3,4}, 3,2 \mathrm{~Hz}$; $J_{3,2}, 10,4 \mathrm{~Hz} ; \mathrm{H}-3$ '); 4,82 (t; $\left.J_{2,1}=J_{2,3} 9,1 \mathrm{~Hz} ; \mathrm{H}-2\right) ; 4,76\left(\mathrm{t} ; J_{1, \mathrm{NH}}=J_{1,2}\right.$ $9,1 \mathrm{~Hz} ; \mathrm{H}-1) ; 4,59$ (dd; $\left.J_{6 \mathrm{a}, 5} 1,7 \mathrm{~Hz} ; J_{6 \mathrm{a}, 6 \mathrm{~b}} 10,4 \mathrm{~Hz} ; \mathrm{H}-6 \mathrm{a}\right) ; 4,51$ (d; $J_{1,2}$, 7,9 Hz; H-1'); 4,14 (dd; $J_{6 \mathrm{~b}}, 5,6,6 \mathrm{~Hz} ; J_{6 \mathrm{~b}}, 6 \mathrm{a}, 11,1 \mathrm{~Hz} ; \mathrm{H}-6 \mathrm{~b}$ '); 4,114,05 (m; H-6b e H-6a'); 3,89 (t; $J_{5,6}, 6,6$ Hz; H-5'); 3,76-3,67 (m; H4 e H-5); 3,05 (s; H-7); 2,15 (s; OCOC $\left.\underline{H}_{3}\right) ; 2,11$ (s; OCOC $\left.\underline{H}_{3}\right) ; 2,08$ $\left(\mathrm{s} ; \mathrm{OCOC}_{3}\right) ; 2,06\left(\mathrm{~s} ; \mathrm{OCOC}_{3}\right) ; 2,05\left(\mathrm{~s} ; \mathrm{OCOC}_{3}\right) ; 2,04(\mathrm{~s} ;$ OCOC $\left.\underline{H}_{3}\right) ; 1,97\left(\mathrm{~s} ; \mathrm{OCOC}_{3}\right)$. RMN de ${ }^{13} \mathrm{C}\left(\delta ; \mathrm{CDCl}_{3} ; 100 \mathrm{MHz}\right)$ : $170,99,170,37,170,16,170,13,170,09,169,30,168,95$ (7C; $\mathrm{OCOCH}_{3}$ ); 100,89 (C-1'); 82,71 (C-1); 75,99 (C-4); 74,45 (C-5); 72,23 (C-3); 70,95; 70,82 (C-3' e C-5'); 70,23 (C-2); 69,13 (C-2'); 66,66 (C-4'); 61,19 (C-6); 60,87 (C-6'); 43,55 (C-7); 2, 15, 2,11, 2,08, 2,06, 2,05, 2,04, 1,97 (7C; $\left.\mathrm{OCOCH}_{3}\right)$.

\section{2,3,6-tri-O-acetil-4-O-(2,3,4,6-tetra-O-acetil- $\beta$ - $D$ -} galactopiranosil)-1-benzenossufonilamino-1-desoxi- $\beta-D-$ glicopiranose (12d)

O material bruto obtido da síntese da sulfonamida 12d foi purificado por cromatografia em coluna de sílica (clorofórmio/metanol 98:2). A partir de $489 \mathrm{mg}$ do material bruto foram isolados $105 \mathrm{mg}$ do anômero $\beta$ 12c (0,13 mmol; $19 \%$ a partir de 8). Tf: 105,5-106,1 ${ }^{\circ} \mathrm{C} .[\alpha]_{\mathrm{D}}+14,8\left(c 2, \mathrm{CHCl}_{3}\right)$. IV $\left(v_{\mathrm{mix}}, \mathrm{cm}^{-1}\right): 1744,1437,1367,1218$, 1039. $\mathrm{RMN}$ de ${ }^{1} \mathrm{H}\left(\delta ; \mathrm{CDCl}_{3} ; 400 \mathrm{MHz}\right): 7,88-7,86$ (m; H-8 e H-8');

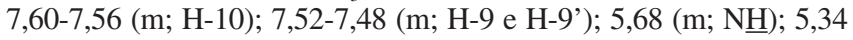
(dd; $J_{4,5}, 0,9 \mathrm{~Hz} ; J_{4,3}, 3,4 \mathrm{~Hz} ; \mathrm{H}-4$ '); 5,25 (m; H-3); 5,09 (dd; $J_{2,1}, 7,8$ Hz; $J_{2,3}, 10,4$ Hz; H-2'); 4,95 (dd; $J_{3,4}, 3,4$ Hz; $J_{3,2}, 10,4$ Hz; H-3'); 4,81-4,75 (m; H-1 e H-2); 4,44 (d; $J_{1,2}, 7,8$ Hz; H-1'); 4,30 (dd; $J_{6 \mathrm{a}, 5}$ $\left.1,9 \mathrm{~Hz} ; J_{6 \mathrm{a}, 6 \mathrm{~b}} 12,0 \mathrm{~Hz} ; \mathrm{H}-6 \mathrm{a}\right) ; 4,13$ (dd; $J_{6 \mathrm{a}^{\prime}, 6 \mathrm{~b}^{\prime}}, 6,4 \mathrm{~Hz} ; J_{6 \mathrm{a}^{\prime}, 6 \mathrm{~b}^{\prime}}, 11,1 \mathrm{~Hz}$; H-6a'); 4,07 (dd; $J_{6 b^{\prime}, 5}, 7,2 \mathrm{~Hz} ; J_{6 b^{\prime}, 6 a^{\prime}} 11,1 \mathrm{~Hz} ; \mathrm{H}-6 \mathrm{~b}$ '); 4,00 (dd; $J_{6 \mathrm{~b}, 5}$ $\left.4,8 \mathrm{~Hz} ; J_{6 \mathrm{~b}, 6 \mathrm{a}} 12,0 \mathrm{~Hz} ; \mathrm{H}-6 \mathrm{~b}\right) ; 3,86$ (tl; $J_{5,4}, 0,9 \mathrm{~Hz} ; J_{5,6 \mathrm{~b}}, 7,2 \mathrm{~Hz} ; \mathrm{H}-$ 5'); 3,71 (t; $\left.J_{4,3}=J_{4,5} 9,9 \mathrm{~Hz} ; \mathrm{H}-4\right) ; 3,62$ (ddd; $J_{5,6 \mathrm{a}} 1,9 \mathrm{~Hz} ; J_{5,6 \mathrm{~b}} 4,8 \mathrm{~Hz}$; $\left.J_{5,4} 9,9 \mathrm{~Hz} ; \mathrm{H}-5\right) ; 2,15\left(\mathrm{~s} ; \mathrm{OCOC}_{3}\right) ; 2,07\left(\mathrm{~s} ; \mathrm{OCOCH}_{3}\right) ; 2,06(\mathrm{~s} ;$ OCOC $\left.\underline{H}_{3}\right) ; 2,05\left(\mathrm{~s} ; \mathrm{OCOC}_{3}\right) ; 2,04\left(\mathrm{~s} ; \mathrm{OCOC} \underline{H}_{3}\right) ; 1,99\left(\mathrm{~s} ; \mathrm{OCOC}_{3}\right)$; $1,94\left(\mathrm{~s} ; \mathrm{OCOCH}+\underline{\mathrm{H}}_{3}\right) . \mathrm{RMN} \mathrm{de}{ }^{13} \mathrm{C}\left(\delta ; \mathrm{CDCl}_{3} ; 100 \mathrm{MHz}\right): 170,87,170,33$, $170,12,170,10,170,04,169,34,168,95\left(7 \mathrm{C} ; \mathrm{OCOCH}_{3}\right) ; 141,28(\mathrm{C}-$ 7); 132,86 (C-10); 128,94 (C-8); 126,96 (C-9); 100,81 (C-1'); 82,62 (C-1); 75,69 (C-4); 74,35 (C-5); 72,25 (C-3); 70,94; 70,75 (C-3' е C5'); 70,49 (C-2); 69,06 (C-2'); 66,65 (C-4'); 61,67 (C-6); 60,67 (C6'); 20,76, 20,68, 20,58, 20,45 (7C; $\left.\mathrm{OCOCH}_{3}\right)$.

\section{Síntese de 13a-e e 14a-d: procedimento geral}

Em balão de $100 \mathrm{~mL}$, imerso em banho de gelo, os derivados per- $O$-acetilados foram dissolvidos em $20 \mathrm{~mL}$ de solução metanólica de metóxido de sódio, preparada pela dissolução de um pequeno cubo de sódio metálico (entre 100-150 mg) em metanol anidro. A mistura reagente foi deixada à temperatura ambiente e sob agitação magnética por 60-90 min. Ao término da reação, a solução metanólica foi neutralizada com resina de troca catiônica Amberlite IRA-120. A resina foi removida por filtração e o solvente destilado em evaporador rotatório. Quando necessário, o sólido obtido foi recristalizado em solvente apropriado. O tempo de reação, rendimento e os solventes utilizados na recristalização dos produtos desacetilados são mostrados na Tabela 2 .

\section{1-acetilamino-1-desoxi- $\beta$-D-galactopiranose (13a)}

A partir de $1 \mathrm{~g}$ de 11a $(2,56 \mathrm{mmol})$, foram obtidos $556 \mathrm{mg}$ de 13a (2,50 mmol; 98\%). Sólido branco. Tf: $234,1-235,2{ }^{\circ} \mathrm{C}$ (lit.: $\left.233{ }^{\circ} \mathrm{C}^{38}\right) \cdot[\alpha]_{\mathrm{D}}+8,0\left(c 2, \mathrm{H}_{2} \mathrm{O}\right)\left(\right.$ lit.: $\left.+9,8, c 2, \mathrm{H}_{2} \mathrm{O}^{38}\right)$. IV $\left(\mathrm{v}_{\max }, \mathrm{cm}^{-}\right.$ $\left.{ }^{1}\right): 3383,3311,3270,1670,1548,1084,1044$. RMN de ${ }^{1} \mathrm{H}(\delta$; DMSO; $200 \mathrm{MHz}): 8,36\left(\mathrm{~d} ; J_{\mathrm{NH}, 1} 9,1 \mathrm{~Hz} ; \mathrm{N} \underline{\mathrm{H}}\right) ; 4,76$ (d; $J$ 4;2 Hz; $\mathrm{OH}) ; 4,69-4,53(\mathrm{~m} ; 5 \mathrm{H}) ; 4,4$ (d; $J 3 ; 2 \mathrm{~Hz} ; \mathrm{OH}) ; 3,66(\mathrm{sl} ; 1 \mathrm{H}) ; 3,45-$ $3,33$ (m; $5 \mathrm{H}) ; 1,81\left(\mathrm{~s} ; \mathrm{COC}_{3}\right)$. RMN de ${ }^{13} \mathrm{C}(\delta$; DMSO; $50 \mathrm{MHz})$ : $169,78\left(\mathrm{COCH}_{3}\right) ; 79,94(\mathrm{C}-1) ; 76,64(\mathrm{C}-3) ; 74,23$ (C-5); 69,70 (C2); 68,28 (C-4); 60,54 (C-6); 22,92 ( $\left.\mathrm{COCH}_{3}\right)$.

\section{1-benzoilamino-1-desoxi- $\beta$-D-galactopiranose (13b)}

A partir de 1,7 g de 11b (3,76 mmol), foram obtidos $1,01 \mathrm{~g}$ de 13b (3,60 mmol; 95\%). Sólido branco. Tf: $117-119{ }^{\circ} \mathrm{C}$ (lit.: $114-118$ $\left.{ }^{\circ} \mathrm{C}^{37}\right) .[\alpha]_{\mathrm{D}}+38,0\left(c 2, \mathrm{H}_{2} \mathrm{O}\right)\left(\right.$ lit.: $\left.+25,0, c 0,02, \mathrm{H}_{2} \mathrm{O}^{39}\right)$. IV $\left(\mathrm{v}_{\text {máx }}, \mathrm{cm}^{-}\right.$ $\left.{ }^{1}\right): 3305,1639,1602,1578,1542,1492,1448,1077 . \mathrm{RMN}$ de ${ }^{1} \mathrm{H}(\delta$; DMSO; $200 \mathrm{MHz}): 8,86$ (d; $\left.J_{\mathrm{NH}, 1} 8,8 \mathrm{~Hz} ; \mathrm{NH}\right) ; 7,93$ (d; $J_{8,9} 6,6 \mathrm{~Hz}$; H-8); 7,53-7,42 (m; H-9 e H-10); 4,94 (t; $\left.J_{1, \mathrm{NH}} 8,9 \mathrm{~Hz} ; \mathrm{H}-1\right) ; 4,81$ (sl; 1H); 4,62 (sl; 1H); 4,45 (sl; 1H); 3,74-3,46 (m; H-5; H-6a e H-6b). RMN de ${ }^{13} \mathrm{C}$ ( $\delta$; DMSO; $\left.50 \mathrm{MHz}\right): 166,79$ (NHCO); 134,21 (C-7); 131,53 (C-10); 128,24 (C-8); 127,73 (C-9); 80,88 (C-1); 76,99 (C3); 74,33 (C-5); 69,43 (C-2); 68,45 (C-4); 60,66 (C-6).

\section{1-desoxi-1-metanossulfonilamino- $\beta$-D-galactopiranose (13c)}

A partir de $1,2 \mathrm{~g}$ de 11c $(2,82 \mathrm{mmol})$, foram obtidos $630 \mathrm{mg}$ de 13c $\left(2,4\right.$ mmol; 87\%). Sólido branco. Tf: $97,5-98,5^{\circ} \mathrm{C} .[\alpha]_{\mathrm{D}}+14,1$ (c 1; $\left.\mathrm{CH}_{3} \mathrm{OH}\right)$. IV $\left(v_{\text {mix }}, \mathrm{cm}^{-1}\right): 3542,3316,3125,1302,1142,1035$. RMN de ${ }^{1} \mathrm{H}(\delta$; DMSO; $200 \mathrm{MHz}): 7,94\left(\mathrm{~d} ; J_{\mathrm{NH}, 1} 7,4 \mathrm{~Hz} ; \mathrm{N} \underline{\mathrm{H}}\right)$; 4,86-4,79 (m; 2H); 4,58 (t; J 5,2 Hz; H-4); 4,45 (d; $1 \mathrm{H} ; J 33,34 \mathrm{~Hz})$; $4,18(\mathrm{tl} ; 1 \mathrm{H}) ; 3,47-3,32(\mathrm{~m} ; 6 \mathrm{H}) ; 2,95\left(\mathrm{~s} ; 3 \mathrm{H} ; \mathrm{SO}_{2} \underline{\mathrm{H}}_{3}\right)$. RMN de ${ }^{13} \mathrm{C}(\delta$; DMSO; $50 \mathrm{MHz}): 85,33(\mathrm{C}-1) ; 76,68(\mathrm{C}-3) ; 74,19(\mathrm{C}-5)$; 69,48 (C-2); 68,26 (C-4); 60,69 (C6); 43,43 ( $\mathrm{SO}_{2} \underline{\mathrm{C}} \mathrm{H}_{3}$ ).

\section{1-benzenossulfonilamino-1-desoxi- $\beta$-D-galactopiranose (13d)}

A partir de 1,6 g de 11d (3,28 mmol), foram obtidos $960 \mathrm{mg}$ de 13d (3,00 mmol; 92\%), após recristalização com isopropanol. Sólido branco cristalino. Tf: $143,5-145,0^{\circ} \mathrm{C}$. $[\alpha]_{\mathrm{D}}+16,0\left(c 1, \mathrm{CH}_{3} \mathrm{OH}\right)$. IV $\left(v_{\text {máx }}, \mathrm{cm}^{-1}\right): 3380,3228,1584,1477,1459,1448,1335,1152$, 1032. RMN de ${ }^{1} \mathrm{H}(\delta$; DMSO; $200 \mathrm{MHz}): 8,47$ (d; $J_{\mathrm{NH}, 1} 8,96 \mathrm{~Hz}$; $\mathrm{NH}) ; 7,85$ (d; $J_{8.9} 6,8 \mathrm{~Hz}$; H-8); 7,57-7,46 (m; H-9 e H-10); 4,854,77 (m; 2H); 4,40-4,29 (m; 3H); 3,63 (s; 1H); 3,36-3,17 (m; 4H); $3,11-3,04(\mathrm{~m} ; 1 \mathrm{H})$. RMN de ${ }^{13} \mathrm{C}(\delta$; DMSO; $50 \mathrm{MHz}): 143,03(\mathrm{C}-$ 7); 131,94 (C-10); 128,47 (C-8); 126,88 (C-9); 85,32 (C-1); 76,11 (C-3); 74,25 (C-5); 69,39 (C-2); 67,59 (C-4); 59,52 (C-6).

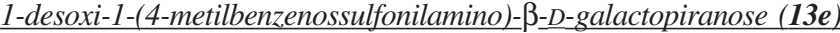

A partir de $1 \mathrm{~g}$ de 11e $(2,00 \mathrm{mmol})$, foram obtidos $600 \mathrm{mg}$ de 13e $(1,80 \mathrm{mmol} ; 91 \%)$. Sólido branco amorfo. Tf: $171,8-173,1^{\circ} \mathrm{C}$. $[\alpha]_{\mathrm{D}}+11,9\left(c 1, \mathrm{CH}_{3} \mathrm{OH}\right)$. IV $\left(v_{\text {máx }}, \mathrm{cm}^{-1}\right): 3411,3246,1629,1599$, $1496,1457,1403,1336,1156,1037,815$. RMN de ${ }^{1} \mathrm{H}(\delta$; DMSO;

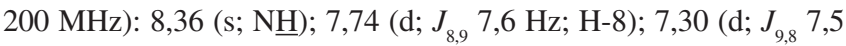
Hz; H-9); 4,78-4,74 (m; 2H); 4,34-4,33 (m; 3H); 3,64 (sl; 1H); 3,36-3,27 (m; 5H); 3,14-3,11 (m; 1H); $2,35\left(\mathrm{~s} ; \mathrm{ArCH}_{3}\right)$. RMN de ${ }^{13} \mathrm{C}(\delta$; DMSO; $50 \mathrm{MHz}): 141,99(\mathrm{C}-7) ; 140,27$ (C-10); 128,84 (C8); 126,94 (C-9); 85,30 (C-1); 76,14 (C-3); 74,24 (C-5); 69,40 (C2); 67,63 (C-4); 59,60 (C-6); 20,97 ( $\left.\mathrm{ArCH}_{3}\right)$. 
1-acetilamino-1-desoxi-4-O-( $\beta$-D-galactopiranosil $)-\beta-\underline{-}-$ glicopiranose $(\mathbf{1 4 a})$

A partir de $680 \mathrm{mg}$ de 12a (1,00 mmol), foram obtidos $213 \mathrm{mg}$ de 14a $(0,55 \mathrm{mmol} ; 60 \%)$, após recristalização com isopropanol/ água 4:1. Cristal incolor. Tf: $158,5-161,5{ }^{\circ} \mathrm{C} ; 237,9-241,5{ }^{\circ} \mathrm{C}$ (o cristal funde a $158,5-161,5{ }^{\circ} \mathrm{C}$, solidifica a $167{ }^{\circ} \mathrm{C}$ e volta a fundir e com decomposição entre $237,9-241,5^{\circ} \mathrm{C}$ ) (lit.: $246-248{ }^{\circ} \mathrm{C}$, com decomposição $\left.0^{40}\right) .[\alpha]_{\mathrm{D}}+1,0\left(c\right.$ 2,5, $\left.\mathrm{H}_{2} \mathrm{O}\right)\left(\right.$ lit.: $\left.[\alpha]_{\mathrm{D}}+1,5, c 1, \mathrm{H}_{2} \mathrm{O}^{40}\right)$. IV $\left(\mathrm{v}_{\text {máx }}, \mathrm{cm}^{-1}\right): 3336,3278,1642,1543$. RMN de ${ }^{1} \mathrm{H}(\delta ; \mathrm{DMSO}$; $400 \mathrm{MHz}): 8,40$ (d; $\left.J_{\mathrm{NH}, 1} 9,0 \mathrm{~Hz} ; \mathrm{N} \underline{\mathrm{H}}\right) ; 5,08$ (d; $J$ 4,0 Hz; O프); 5,00 $(\mathrm{d} ; J 5,3 \mathrm{~Hz} ; \mathrm{OH}) ; 4,75-4,70(\mathrm{~m} ; \mathrm{O} \underline{\mathrm{H}}) ; 4,71\left(\mathrm{~d} ; J_{1, \mathrm{NH}}=J_{1,2} 9,0 \mathrm{~Hz} ; \mathrm{H}-\right.$ 1); 4,64 (t; $J$ 4,9 Hz; OH) ; 4,52 (t; J 5,9 Hz; OH); 4,49 (d; J 4,5 Hz; $\mathrm{OH}) ; 4,20\left(\mathrm{~d} ; J_{1,2}, 7,0 \mathrm{~Hz} ; \mathrm{H}-1\right.$ '); 3,70 (ddl; $J_{6 \mathrm{a}, 5} 5,4 \mathrm{~Hz} ; J_{6 \mathrm{a}, 6 \mathrm{~b}} 11,4$ $\mathrm{Hz}$; H-6a); 3,61-3,44 (m; $5 \mathrm{H}) ; 3,31$ (sl; 1H); 3,11 (dt; $J_{2, \mathrm{OH}} 5,60$ $\left.\mathrm{Hz} ; J_{2,1}=J_{3,3} 8,8 \mathrm{~Hz} ; \mathrm{H}-2\right) ; 1,84\left(\mathrm{~s} ; \mathrm{NHCOC}_{3}\right) . \mathrm{RMN} \mathrm{de}{ }^{13} \mathrm{C}(\delta$, DMSO, $100 \mathrm{MHz})$ : 169,86 ( $\left.\mathrm{NHCOCH}_{3}\right) ; 103,79$ (C-1'); 80,56 (C4); 79,23 (C-1); 76,41, 75,71, 75,54 (C-2, C-3, C-5 e C-5'); 73,23 (C-2' ou C-3'); 72,13 (C-2); 70,58 (C-2' ou C-3'); 68,18 (C-4'); 60,45 e 60,36 (C-6 e C-6'); 22,85 (C-7).

\section{1-benzoilamino-1-desoxi-4-O-( $\beta$-D-galactopiranosil)- $\beta$ - $D$ - glicopiranose $(\mathbf{1 4 b})^{41}$}

A partir de $410 \mathrm{mg}$ de $\mathbf{1 2 b}(0,55 \mathrm{mmol})$, foram obtidos $185 \mathrm{mg}$ de 14b $(0,42 \mathrm{mmol} ; 76 \%)$, após recristalização com metanol. Sólido branco. Decompõe-se, sem fundir, a $211,7{ }^{\circ} \mathrm{C}$. $[\alpha]_{\mathrm{D}}+1,0(c$ 2,5, $\left.\mathrm{H}_{2} \mathrm{O}\right)$. IV $\left(\mathrm{v}_{\text {máx }}, \mathrm{cm}^{-1}\right): 3551,3467,3368,3254,1655,1538$. RMN de ${ }^{1} \mathrm{H}(\delta$, DMSO, $400 \mathrm{MHz}): 8,87\left(\mathrm{~d} ; J_{\mathrm{NH}, 1} 8,7 \mathrm{~Hz} ; \mathrm{NH}\right) ; 7,91(\mathrm{~d}$; $J_{8,10} 7,4 \mathrm{~Hz} ; \mathrm{H}-8$ e H-8'); 7,56 (m; H-10); 7,48 (t; $J_{9,8}=J_{9,10} 7,4 \mathrm{~Hz}$;

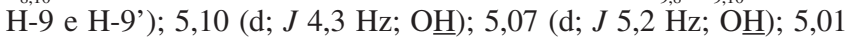
(tl; $\left.J_{1, \mathrm{NH}}=J_{1,2} 8,7 \mathrm{~Hz} ; \mathrm{H}-1\right) ; 4,76(2 \mathrm{~d} ; \mathrm{OH}(2 \mathrm{H})) ; 4,66$ (t; J 5,0 Hz; $\mathrm{OH}) ; 4,55(\mathrm{t} ; J$ 6,0 Hz; O$) ; 4,50(\mathrm{~d} ; J 4,6 \mathrm{~Hz} ; \mathrm{OH}) ; 4,25\left(\mathrm{~d} ; J_{1^{\prime}, 2}\right.$, 7,0 Hz; H-1'); 3,75 (ddl; $\left.J_{6 \mathrm{a}, 5} 5,6 ; J_{6 \mathrm{a}, 6 \mathrm{~b}} 11,0 \mathrm{~Hz} ; \mathrm{H}-6 \mathrm{a}\right) ; 3,65-3,35$ (m; $11 \mathrm{H}) . \mathrm{RMN}$ de ${ }^{13} \mathrm{C}(\delta$; DMSO; $100 \mathrm{MHz}): 166,76\left(\mathrm{NHCOC}_{6} \mathrm{H}_{5}\right)$; 134,05 (C-7); 131,51 (C-10); 128,20 (C-8); 127,57 (C-9); 103,81 (C-1'); 80,69 (C-1); 80,09 (C-4); 76,66, 75,78, 75,53 (C-2, C-3, C5 e C-5'); 73,23 (C-2' ou C-3'); 71,67 (C-2); 70,89 (C-2' ou C-3'); 68,18 (C-4'); 60,44 (C-6 e C-6').

\section{1-desoxi-4-O-( $\beta$-D-galactopiranosil $)-1$-metanossulfonilamino- $\beta=$ D-glicopiranose $(\mathbf{1 4 c})$}

A partir de $350 \mathrm{mg}$ de $12 \mathrm{c}(0,49 \mathrm{mmol})$, foram obtidos $101 \mathrm{mg}$ de 14c $(0,24$ mmol; 49\%), após recristalização com metanol. Sólido branco. Tf: $169,4-171,0{ }^{\circ} \mathrm{C} .[\alpha]_{\mathrm{D}}+10,47\left(c 0,53, \mathrm{H}_{2} \mathrm{O}\right)$. IV $\left(v_{\max }\right.$, $\left.\mathrm{cm}^{-1}\right): 3359,3246,1462,1317,782 . \mathrm{RMN}$ de ${ }^{1} \mathrm{H}(\delta, \mathrm{DMSO}, 400$ $\mathrm{MHz}): 8,05$ (d; $\left.J_{\mathrm{NH}, 1} 8,8 \mathrm{~Hz} ; \underline{\mathrm{H}}\right) ; 5,14$ (d; J 5,8 Hz; O프); 5,07 (d; $J 3,8 \mathrm{~Hz} ; \mathrm{OH}) ; 4,74(\mathrm{~s} ; \mathrm{OH}(2 \mathrm{H})) ; 4,64(\mathrm{t} ; J 5,0 \mathrm{~Hz} ; \mathrm{OH}) ; 4,54(\mathrm{t} ; J$ $5,6 \mathrm{~Hz} ; \mathrm{OH}) ; 4,52(\mathrm{~d} ; J 4,5 \mathrm{~Hz} ; \mathrm{OH}) ; 4,28\left(\mathrm{tl} ; J_{1, \mathrm{NH}}=J_{1,2} 8,8 \mathrm{~Hz} ; \mathrm{H}-\right.$ 1); 4,20 (d; $J_{1,2}, 6,6 \mathrm{~Hz} ; \mathrm{H}-1$ '); 3,79 (ddl; $J_{6 \mathrm{a}, 5} 4,5 \mathrm{~Hz} ; J_{6 \mathrm{a}, 6 \mathrm{~b}} 10,8 \mathrm{~Hz}$; H-6a); 3,61 (sl; H-4'); 3,56-3,40 (m; 4H); 3,20 (dd; $J_{6,5}$ ou $J_{6,5}, 4,5$ $\mathrm{Hz} ; J_{\text {gem }} 10,8 \mathrm{~Hz}$; H-6b ou H-6a'); 3,12 (dt; $J_{2, \mathrm{OH}} 6,2 \mathrm{~Hz} ; J_{2,1}=J_{2,3} 8,6$ $\mathrm{Hz}$; H-2); 2,97 (s; H-7). RMN de ${ }^{13} \mathrm{C}$ ( $\delta$; DMSO; $\left.100 \mathrm{MHz}\right): 103,77$ (C-1'); 84,45 (C-1); 80,66 (C-4); 76,25 (C-5); 75,68, 75,52 (C-3 e C-5'); 73,24 (C-2' ou C-3'); 72,00 (C-2); 70,52 (C-2' ou C-3'); 68,15 (C-4'); 60,56, 60,40 (C-6 e C-6'); 43,32 (C-7).

\section{1-benzenossufonilamino-1-desoxi-4-O- $(\beta$ - $D$-galactopiranosil $)-\beta_{-}$ D-glicopiranose $(\mathbf{1 4 d})$}

A partir de $450 \mathrm{mg}$ de $\mathbf{1 2 d}(0,58 \mathrm{mmol})$, foram obtidos $200 \mathrm{mg}$ de 14d (0,31 mmol; 53\%), após recristalização com metanol. Cristal branco. Tf: $244,9-245,8{ }^{\circ} \mathrm{C}$. $[\alpha]_{\mathrm{D}}+37,7$ (c 1,25, $\left.\mathrm{H}_{2} \mathrm{O}\right)$. RMN de ${ }^{1} \mathrm{H}(\delta ; \mathrm{DMSO} ; 400 \mathrm{MHz}): 8,52(\mathrm{sl} ; \mathrm{N} \underline{\mathrm{H}}) ; 7,83$ (d; $J_{8.9} 7,3 \mathrm{~Hz} ; \mathrm{H}-8$ e H-8'); 7,57 (t; $\left.J_{10.9} 7,3 \mathrm{~Hz} ; \mathrm{H}-10\right) ; 7,50$ (t; $J_{9,8}=J_{9,10} 7,3 \mathrm{~Hz} ; \mathrm{H}-9$ e H-

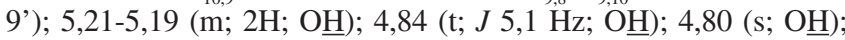

4,78 (d; J 1,4 Hz; OH); 4,57 (d; J 4,7 Hz; OH); 4,39-4,34 (m; 2H; $\mathrm{OH}) ; 4,17$ (m; H-1); 3,81-3,68 (m; H-1',); 3,60 (d; $J_{4}, 3,3,6 \mathrm{~Hz} ; \mathrm{H}-$ 4'); 3,51-3,20 (m; 7H); 3,09 (m; H-2). RMN de ${ }^{13} \mathrm{C}(\delta ; \mathrm{DMSO} ; 100$ MHz): 142,60 (C-7); 133,76 (C-10); 129,96 (C-8); 127,66 (C-9); 104,10 (C-1'); 85,17 (C-1); 79,74 (C-4); 76,44, 76,29, 73,64, 72,64, 71,54, 71,10 (C-2, C-3, C-5, C-2', C-3' e C-5'); 69,20 (C-4'); 61,60 (C-6); 60,70 (C-6')

\section{Ensaio de inibição de hemaglutinação ${ }^{25}$}

Os ensaios de hemaglutinação foram realizados em poços de placa de microtitulação em um volume final de $150 \mu \mathrm{L}$. Foram adicionados, em poços diferentes, $50 \mu \mathrm{L}$ de solução dos carboidratos 1 e 2 e de seus derivados 13a-e e 14a-d em diluições seriadas 1:2 a partir de $0,2 \mathrm{~mol} / \mathrm{L}$. Em cada poço foram adicionados $50 \mu \mathrm{L}$ de solução de lectina, com a menor concentração capaz de promover hemaglutinação. Após 30 min, à temperatura ambiente, foram adicionados $50 \mu \mathrm{L}$ de suspensão de eritrócitos tipo O humanos $4 \% \mathrm{v} / \mathrm{v}$, em $0,15 \mathrm{~mol} / \mathrm{L}$ de cloreto de sódio, $5 \mathrm{mmol} / \mathrm{L}$ de cloreto de cálcio e 5 $\mathrm{mmol} / \mathrm{L}$ de cloreto de manganês. Após $1 \mathrm{~h}$, à temperatura ambiente, foi feita a leitura da hemaglutinação a olho nu (considera-se a menor concentração capaz de inibir a hemaglutinação). O experimento foi realizado em triplicata. A atividade inibitória da hemaglutinação mediada por lectina foi expressa em valores de inibição relativa à inibição promovida pela D-galactose.

\section{REFERÊNCIAS}

1. Liener, I. E.; Sharon, N.; Goldstein, I. J.; The Lectin: Properties, Functions, and Applications in Biology and Medicine, Orlando:Academic Press, Inc., 1986.

2. Sharon, N.; Lis, H.; Science 1989, $246,227$.

3. Loris, R.; Biochim. Biophys. Acta 2002, 1572, 198.

4. Rüdiger, H.; Gabius H. J.; Glycoconjugate J. 2001, 18, 589

5. Lis, H.; Sharon, N.; Chem. Rev. 1998, 98, 637.

6. Sharon, N.; Lis, H.; Glycobiology 2005, 14, 53R

7. Pyz, E.; Marshall, A. S. J.; Gordon, S.; Brown, G. D.; Ann. Med. 2006, 38, 242.

8. Pieters, R. J.; Chembiochem. 2006, 7, 721.

9. Taylor, M.; Drickamer, K.; Introduction to Glycobiology, Oxford University Press, 2003.

10. Corbell J. B.; Lundquist, J. J.; Toone, E. J.; Tetrahedron: Asimmetry. 2000, 11,95 .

11. http://www.cermav.cnrs.fr/lectins, acessada em Agosto 2006.

12. Svensson, C.; Teneberg, S.; Nilsson, C. L.; Kjellberg, A.; Schwarz, N.; Krengel, U.; J. Mol. Biol. 2002, 321, 69.

13. Iglesias, J. 1.; Lis, H.; Sharon, N.; Eur. J. Biochem. 1982, 123, 247.

14. Turton, K.; Natesh, R.; Thiyagarajan, N.; Chaddock, J. A.; Acharya, K. R.; Glycobiology 2004, 14, 923.

15. Solís, D.; Fernández, P.; Díaz-Mauriño, T.; Jiménez-Barbero, J.; MartinLomas, M.; Eur. J. Biochem. 1993, 214, 677.

16. Peacock, J. S.; Colsky, A. S.; Pinto, V. B.; J. Immun. Methods 1990, 126, 140.

17. Gabius, H. J.; Pharm. Res. 1998, 15, 23.

18. Smiataczowa, K.; Kosmalski, J.; Nowacki, A.; Czaja, M.; Warnke, Z.; Carbohydr. Res. 2004, 339, 1439.

19. Hudson, C. S.; Kuns, A.; J. Am. Chem. Soc. 1925, 47, 2052.

20. Conchie, J.; Levvy, G. A.; Marsch, C. A.; Adv. Carbohydr. Chem. 1957, 12,157

21. Tropper, F. D.; Anderson, F. O.; Braum, S.; Roy, R.; Synthesis 1992, 618.

22. Sinnott, M. L.; Biochem. J. 1971, 125, 717.

23. Lubineau, A.; Augé, J.; Drouillat, B.; Carbohydr. Res. 1995, 266, 211

24. Czifrak, K.; Hadady, Z.; Docsa, T.; Gergely, P.; Schmidt, J.; Wessjohann, L.; Somsak, L.; Carbohyd. Res. 2006, 341, 947.

25. Konozy, E. H.; Bernardes, E. S.; Rosa, C.; Faça, V.; Greene, L. J.; Ward, R. J.; Arch. Biochem. Biophys. 2003, 410, 222.

26. Svensson, C.; Teneberg, S.; Nilsson, C. L.; Kjellberg, A.; Schwarz, F. P.; Sharon, N.; Krengel, U.; J. Mol. Biol. 2002, 321, 69.

27. Rutenber, E.; Katzin, B. J.; Ernst, S.; Collins, E. J.; Mlsna, D.; Ready, M. P.; Robertus, J. D.; Proteins 1991, 10, 240. 
28. Dawson, R. M.; Alderton, M. R.; Wells, D.; Hartley, P. G.; J. Appl. Toxicol. 2006, 26, 247.

29. Lis, H.; Joubert, F. J.; Sharon, N.; Phytochemistry 1985, 34, 2803; Lin, T. T. S.; Li, Steven S. L.; Eur. J. Biochem. 1980, 105, 453.

30. Jarrahpour, A. A.; Shekarriz, M.; Taslimi, A.; Molecules 2004, 9, 29.

31. Sabesan, S.; Neira, S.; Carbohyd. Res. 1992, 223, 169.

32. Zhang, F. X.; Kuang, D. Z.; Feng, Y. L.; Wang, J. Q.; Xu, Z. F.; Youji Hиахие 2003, 23, 361 .

33. Kamitakahara, H.; Cellulose (Dordrecht, Netherlands) 2005, 12, 209.

34. Peto, C.; Lieb. Ann. Chem. 1991, 5, 505.

35. Maier, M. A.; Yannopoulos, C. G.; Mohamed, N.; Roland, A.; Fritz, H.; Mohan, V.; Just, G.; Manoharan, M.; Bioconjugate Chem. 2003, 14, 18; Sansone, F.; Chierici, E.; Casnati, A.; Ungaro, R.; Org. Biomol. Chem. 2003, $1,1802$.
36. Frush, H. L.; Isbell, H. S.; J. Res. Natl. Bur. Standards 1951, 47, 239.

37. Kovács, L.; Psz, E.; Domokos, V.; Holzer, W.; Györgydeák, Z.; Tetrahedron 2001, 57, 4609.

38. Priya, K.; Loganathan, D.; Tetrahedron 1999, 55, 1119.

39. Savel'ev, A. N.; Ibatyllin, F. M.; Eneyskaya, E. V.; Kachurin A, M.; Neustroev, K. N.; Carbohydr. Res. 1996, 296, 261.

40. Kuhn, R.; Krüger, G.; Chem. Ber. 1954, 87, 1544.

41. Deferrari, J. O.; Thiel, I. M. E.; Cadenas, R. A.; Carbohydr. Res. 1973 29, 141. Estes autores descrevem a obtenção desta benzamida, sem a especificação de sua configuração anomérica. A benzamida foi descrita como um sólido amorfo higroscópico, com $\left[\alpha_{\mathrm{D}}+63,3\left(c 0,9, \mathrm{H}_{2} \mathrm{O}\right)\right.$. 